\title{
Uso de recursos de información electrónicos 2003-2004
}

\author{
Por Concha Álvaro, Tomàs Baiget, Elea Giménez, Alice Keefer, Dirk Lens, Juan Carlos Martín y Carlos Tejada
}

Resumen: Datos generales de un estudio llevado a cabo en España -y promovido por la agencia de suscripciones Swets Information Services-sobre el uso e implantación de revistas, bases de datos y otros recursos de información electrónicos. Los resultados muestran una ya notable aceptación del soporte electrónico, aunque el papel sigue teniendo sus partidarios principalmente por razones de conservación a largo plazo y facilidad de lectura.

Descriptores: Recursos electrónicos, Revistas, Bases de datos, Preferencias de los usuarios, Modelos de tarificación.

\section{Title: Electronic information resources usage 2003-2004}

Abstract: Overview of a study carried out in Spain - under the auspices of the subscription agency Swets Information Services-on the introduction and use of e-journals, databases and other digital information resources. The results show a high level of acceptance of electronic media, even though paper continues to have strong supporters due mainly to the possibilities of long-term conservation and ease of reading.

Keywords: Electronic resources, Journals, Databases, User preferences, Charging models.

\section{Introducción}

Siguiendo la metodología de una encuesta similar realizada conjuntamente en 2002 por SwetsBlackwell (nombre anterior de Swets Information Services), el Group Romand de Documentation y la revista francesa Archimag a los bibliotecarios de Francia y Suiza (zona francófona), y ante la buena acogida y la obvia utilidad de sus resultados, Swets Information Services decidió actualizarla y, al mismo tiempo, incorporar en la nueva edición a Bélgica y España.

En nuestro país, además de Swets se encargaron de llevarla a cabo la Sociedad Española de Documentación e Información Científica (Sedic) y la revista El profesional de la información (EPI).

En este artículo presentamos un resumen de los resultados españoles.

\section{Población encuestada}

El anuncio y las instrucciones para participar en el estudio se distribuyeron por diferentes canales: envío postal a los socios de Sedic, a los clientes de Swets Information Services y a los suscriptores de $E P I$, así como a través de las listas electrónicas IweTel y Fidel.

En todos los casos se rogaba rellenar el cuestionario electrónico

que estaba colgado en la página web de Swets en 5 idiomas: alemán, español, flamenco, francés e inglés.

\section{«El soporte papel se mantiene porqué los usuarios lo prefieren y por las escasas ga- rantías que ofrecen los editores de per- durabilidad de las co- lecciones digitales»}

El periodo para cumplimentarlo se extendió desde mediados de diciembre de 2003 hasta mediados de enero de 2004. Durante ese tiempo en España se recibieron 262 .

\section{Perfil institucional}

El $67 \%$ de las respuestas son del sector público y el $33 \%$ del privado. Las organizaciones representadas en su mayoría eran grandes, pues el $72 \%$ tenía más de 250 empleados.

Perfil del profesional de la información

Predominan los encargados de la biblioteca o del servicio, con títulos como jefes, directores, subdirectores y responsables, y bastantes años de experiencia.
Un $66 \%$ autocalificaba como "bueno" su conocimiento de internet; el 34\% restante, como "mediano".

\section{Datos cuantitativos de productos electrónicos en las colecciones}

\section{Bases de datos}

Se observan dos grupos mayoritarios: un 19\% de los encuestados dice acceder a 1-5 bases de datos y un $28 \%$ accede a más de 15 .

Esto permite hacer una primera observación y es que la población que ha respondido tiene un perfil heterogéneo (centros grandes y pequeños, públicos y privados, etc.) $y$, por tanto, se encontrarán datos muy variados, lo cual puede suponer un inconveniente a la hora de tratar de identificar tendencias. Asimismo, al estar delimitando ya un tipo de recurso electrónico (bases de datos) parece más lógico que un $36 \%$ no disponga de acceso a ellas. No obstante, es un porcentaje alto para tratarse de profesionales de la información.

\section{Revistas electrónicas}

Si ha sorprendido el porcentaje de profesionales que no cuentan con acceso a bases de datos, la sorpresa aumenta al hablar de revistas electrónicas ya que, en este caso, un $42 \%$ de los encuestados mani- 


\section{* ¿Necesita una solución ágil, abierta y potente para contenidos multimedia?}
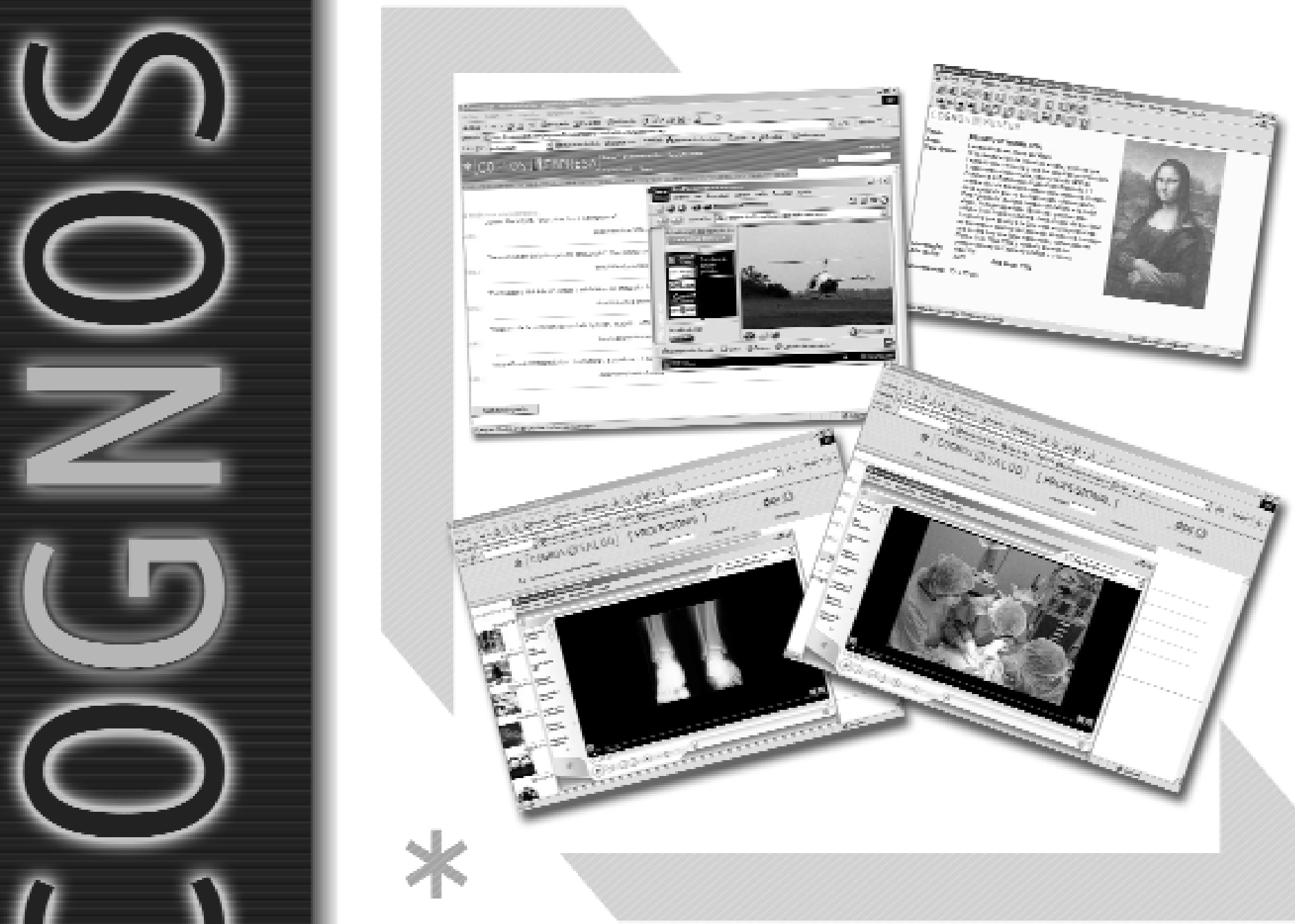

* Con COGNOS puede:

- Almacenar y recuperar contenidos multimedia de manera rápida

- Integrar y mantener los datos asociados a imágenes con facilidad

- Obtener resultados a corto plazo

- Asegurar una fácil implementación

En entorno windows o web

Utilizando un gestor documental o SQL Server

Desarrollado por:

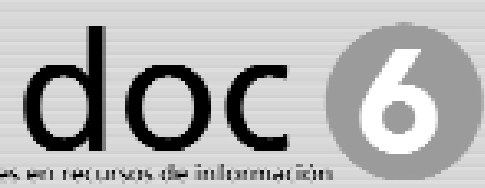

COGNOS ha recibido la eliquela EUREKA de I+D, ha sidto evaluado Cavorablemente porr el CDTI y ha recibida financiacion del Ministerics de Ciencia y Tocnologjia.

Molloras, 272, planta 3
Tol 932154313
Fox 934833621
08037 Bsrcelons

Orenes $14,5^{\circ} \mathrm{A}$ Tol 915535207 Fax 915346112 28020 Modrid 
fiesta no acceder a ellas. Asimismo, la distribución desigual de los datos permite afianzar la tesis de que las personas consultadas trabajan en centros con perfiles muy distintos.

\section{«La importancia de las colecciones en papel sigue siendo muy superior al de las ediciones electró- nicas, aún cuando gran parte de las re- vistas han optado por ofrecer simultá- neamente su versión electrónica»}

Se sigue observando que los conjuntos más numerosos corresponden a los dos extremos. Así, hay un gran grupo (31\%) que accede a 1-50 revistas electrónicas, mientras que el segundo (17\%) accede a más de 500 .

Sólo un 27\% de los profesionales dice que su colección electrónica supone más de un $25 \%$ del total de revistas. Esto permite afirmar que el peso de las colecciones en papel sigue siendo muy superior al de las ediciones electrónicas, aún cuando gran parte de las revistas que se editan en papel han optado por ofrecer simultáneamente su versión electrónica.

Las razones fundamentales del mantenimiento de las colecciones en papel parecen estar, a la vista de otros resultados de este mismo cuestionario, en los propios usuarios que siguen demandando y prefiriendo la lectura en papel y en las escasas garantías de perdurabilidad de las colecciones digitales que están ofreciendo los editores. Ambas razones aparecen repetidas varias veces a lo largo del estudio y se identifican como dos de las grandes preocupaciones de los profesionales de la información españoles.

\section{Bases de datos elaboradas por la propia organización}

Destaca el alto porcentaje de instituciones que elaboran bases de datos propias $(65 \%)$. Este dato puede ser interpretado como una necesidad de estructurar la información interna de la institución con el objetivo de gestionar el conocimiento, agilizar los procesos, mejorar la gestión, etc. Asimismo, teniendo en cuenta las respuestas ofrecidas en otras preguntas del cuestionario, este alto porcentaje también puede deberse a la falta de productos en el mercado adecuados a las necesidades de las instituciones. Más concretamente, cuando los usuarios se pronuncian sobre los retos futuros para los recursos electrónicos, un $50 \%$ de ellos considera muy importante aumentar la oferta dentro de las distintas áreas de especialización. Como se comentará más adelante, esta demanda podría referirse a información especializada o a información de áreas con un fuerte componente local (ciencias humanas y ciencias sociales).

Sí parece evidente que los soportes digitales se imponen frente a los ópticos a la hora de crear bases de datos propias. La posibilidad de "abrir" esas bases de datos a internet a través de pasarelas supone una ventaja añadida.

Los datos son bastante elocuentes ya que un $80 \%$ de las personas que contestaron la pregunta indica que crean bases de datos para las intranets; esto demuestra la extensión de la tecnología dentro de las organizaciones y, sobre todo, su utilización como herra- mienta de apoyo al trabajo diario, a la gestión, etc.

Cerca de un 50\% de las instituciones encuestadas publican bases de datos de producción propia en internet, lo que en principio apunta a un enriquecimiento de la Red en términos informativos.

\section{4.a) Bases de datos referen- ciales}

Entre los que manifestaron acceder a bases de datos, la inmensa mayoría (más del 90\%) consulta bases de datos referenciales. Y lo hace fundamentalmente a través de internet (en más de un $80 \%$ de los casos) y también a través de cdrom, soporte que perdura en la difusión de bases de datos de este tipo: casi un $60 \%$ de los encuestados utiliza este soporte. Una explicación podría estar en la facilidad para compartir estos recursos entre distintas bibliotecas y centros de documentación de un mismo organismo, a través de las cd-nets, en fases previas a la implantación de las correspondientes intranets.

Por otra parte, el porcentaje de instituciones que consultan bases

\begin{tabular}{|l|c|}
\hline \multicolumn{1}{|c|}{ Bases de datos } & $\begin{array}{c}\mathbf{N}^{\circ} \text { veces } \\
\text { citadas }\end{array}$ \\
\hline Medline-PubMed & 29 \\
\hline CSIC-Cindoc & 26 \\
\hline ISI & 20 \\
\hline Westlaw-Aranzadi & 13 \\
\hline Cochrane & 9 \\
\hline ISBN & 8 \\
\hline Psycinfo & 7 \\
\hline Rebiun & 7 \\
\hline La Ley Actualidad & 7 \\
\hline LISA & 6 \\
\hline Embase & 6 \\
\hline Chemical Abstracts & 6 \\
\hline Compludoc & 5 \\
\hline Agricola & 5 \\
\hline Agris & 5 \\
\hline Bibliografia Española & 4 \\
\hline Compendex / Engineering Village & 4 \\
\hline CBUC Sumaris & 3 \\
\hline ASFA & 3 \\
\hline Inspec & 3 \\
\hline ABI Inform & 3 \\
\hline Tirant on line & 2 \\
\hline Biological Abstracts & 2 \\
\hline Cuidatge & 2 \\
\hline
\end{tabular}

Tabla 1. Bases de datos referenciales más utilizadas Base: profesionales que han contestado la pregunta 
de datos de este tipo a través de intranet no es desdeñable (más de un $39 \%$ ), e indica una tendencia hacia la utilización de recursos electrónicos compartidos, que puede apoyarse en otros datos obtenidos de las diferentes preguntas de esta encuesta. Un ejemplo es que los profesionales de la información confieren importancia a la necesidad de integrar recursos electrónicos bajo una misma herramienta; así esta necesidad ocupa el cuarto lugar entre las principales expectativas en relación con los recursos electrónicos.

En la tabla 1 se recogen las bases de datos referenciales más utilizadas. Destaca el hecho de que seis de ellas sean gratuitas (al menos en el momento de hacer la encuesta). De las 223 bases de datos referenciales distintas que se citan, sólo 27 son citadas más de una vez.

\section{4.b) Bases de datos a texto completo}

El primer dato relevante es que la utilización de este tipo de bases de datos se ve reducida en relación con las bases de datos referenciales. Mientras estas últimas eran utilizadas por más de $90 \%$ de los encuestados, las bases de datos a texto completo son utilizadas por algo más del $72 \%$. En ello puede incidir claramente la menor oferta de contenidos íntegros gratuitos, en comparación con los contenidos referenciales.

En cuanto al tipo de soporte se produce una reducción significativa (del $57 \%$ a casi el $40 \%$ ) en la utilización de CD. En este punto hay que considerar que las bases de datos textuales no fueron habituales hasta la irrupción de la tecnología digital que, entre otras cosas, mejora la capacidad de almacenamiento y la actualización. Por ello, también se explica que al hablar de bases de datos a texto completo el soporte internet sea ampliamente utilizado $(77 \%)$.

Por otra parte, las intranets son utilizadas casi en la misma medida para la consulta de bases de datos referenciales (39\%) y bases de datos textuales (32\%). En este caso, hay que considerar que la intranet puede representar una plataforma integradora que da acceso a un recurso compartido internamente o bien puede albergar un simple enlace a un recurso electrónico disponible en internet.

\begin{tabular}{|l|c|}
\hline \multicolumn{1}{|c|}{ Bases de datos de texto completo } & $\begin{array}{c}\mathbf{N}^{\circ} \text { de veces } \\
\text { citadas }\end{array}$ \\
\hline Westlaw-Aranzadi & 28 \\
\hline Iberlex-BOE & 11 \\
\hline PCI Full Text & 10 \\
\hline La Ley & 8 \\
\hline ABI Inform & 5 \\
\hline Business Source Elite & 5 \\
\hline Cochrane & 5 \\
\hline Factiva & 4 \\
\hline Business Source Premier & 3 \\
\hline Celex & 3 \\
\hline Comext & 3 \\
\hline El Derecho & 3 \\
\hline Euromonitor & 3 \\
\hline Iconoce & 3 \\
\hline New Cronos & 3 \\
\hline PubMed & 3 \\
\hline Sabi & 3 \\
\hline Convenios Colectivos de Lex Nova & 2 \\
\hline Diario oficial de la Union Europea & 2 \\
\hline DOIS & 2 \\
\hline ElU & 2 \\
\hline IEL & 2 \\
\hline Maninvest & 2 \\
\hline Suscrinorma AENOR & 2 \\
\hline Tirant Online & 2 \\
\hline & 2 \\
\hline & 2 \\
\hline & 2 \\
\hline & 2 \\
\hline & 2 \\
\hline & 2 \\
\hline
\end{tabular}

Tabla 2. Bases de datos de texto completo más utilizadas Base: profesionales que han contestado la pregunta

También se produce una diferencia significativa en el tipo de suscripciones. Más del $67 \%$ de las bases de datos de texto completo utilizadas por los encuestados son bases de datos de pago, frente al resto que son gratuitas.

\section{«A los profesionales más que el precio les preocupa la claridad de las tarifas que les ofrecen editores $y$ agentes»}

En el caso de las bases de datos a texto completo, los encuestados citan más bases de datos diferentes (hasta un total de 118), entre ellas incluyen las plataformas de editores de revistas o de distribuidores de bases de datos, y no se marcan las diferencias entre aquellas bases de datos para cuya creación se ha llevado a cabo un análisis documental y otras variantes de informaciones estructuradas. Las más utilizadas se muestran en la tabla 2.

\section{Publicaciones electrónicas a texto completo}

El porcentaje de utilización de revistas electrónicas en sus distintas versiones es muy semejante al de utilización de bases de datos referenciales, es decir, supera el $90 \%$. En este caso, tal y como cabría esperar, internet es el escenario protagonista. La expansión de la edición electrónica se produce gracias y a partir de la llegada de internet, por lo cual no extraña el dato de que más del $87 \%$ de las revistas se consulten a través de la Red. Tampoco parece extraño que las intranets alberguen una proporción menor de revistas (cerca del $19 \%$ ) ya que, como instrumento de comunicación interna, trabajo en grupo, etc., es más habitual que incorporen noticias destacadas, comunicados o incluso boletines, que revistas elaboradas por la propia organización; en el caso de organizaciones que requieran acceso a contenidos de revistas la vía más habitual será internet, donde han establecido sus plataformas los grandes editores/distribuidores.

En cuanto a la gratuidad o no de estos productos, sorprende el elevado porcentaje de revistas uti- 


\begin{tabular}{|l|c|}
\hline \multicolumn{1}{|c|}{ Editores } & $\begin{array}{c}\mathrm{N}^{\circ} \text { de veces } \\
\text { citados }\end{array}$ \\
\hline Elsevier & 36 \\
\hline Blackwell & 15 \\
\hline Kluwer & 15 \\
\hline Springer Verlag & 14 \\
\hline Wiley & 13 \\
\hline Doyma & 5 \\
\hline Lippincott Williams \& Wilkins & 5 \\
\hline
\end{tabular}

Tabla 3. Editores más importantes según los profesionales Base: respuestas recibidas

lizadas que son gratuitas $(42,4 \%)$, diez puntos superior al de bases de datos a texto completo gratuitas.

Tres de las posibles causas de esta circunstancia pueden ser:

- la aparición en origen de revistas electrónicas que siendo gratuitas podían competir con la veteranía de otras revistas impresas y, por tanto, cubrir una cierta "cuota de mercado"

—la aparición de los "archivos abiertos" o prepublicaciones, no dependientes de editores, de acceso libre y en plena fase de expansión.

—el acceso a la suscripción electrónica para el usuario cuando éste se ha suscrito previamente a la edición en papel.

Por otra parte, aunque más del $68 \%$ de los profesionales afirma que mantiene la doble suscripción (papel/digital), empieza a ser significativo que haya un $32 \%$ de casos en los que sólo se utiliza la edición electrónica. La facilidad de acceso y de consulta contrarresta la falta de comodidad de la lectura en pantalla, aún no solucionada. De hecho, las reticencias de los profesionales de la información en cuanto a la transformación de su colección a formato digital tienen que ver, por una parte, con los problemas de preservación, pero por otra, con la demanda persistente de los usuarios de las revistas en papel.
Probablemente, en la mayoría de los centros con doble suscripción se esté combinando la utilización de los dos soportes: uno para recuperar y consultar y otro para leer. No obstante, en algunos de los comentarios recogidos en preguntas anteriores, se ha podido apreciar la preocupación de que al precio de la doble suscripción hubieran de añadirse los costes de la impresión en papel de los artículos de revistas electrónicas.

El motivo con más peso para el mantenimiento de la colección en papel es la propia política de los centros de información (49\%). Esta política estará relacionada muy probablemente con los otros dos argumentos que se aportan, es decir, con la demanda de los usuarios (41\%), que siguen pidiendo el soporte papel, y con la necesidad de garantizar la preservación de la colección $(33 \%)$. La política de los editores condiciona en un $31 \%$ el mantenimiento de la colección en papel. El problema de la preservación y de la perdurabilidad de los soportes, así como las dificultades de la lectura en pantalla podrían ser las fronteras del salto definitivo a las colecciones digitales.

Algunos encuestados señalan que los altos costes de la doble suscripción, la falta de espacio para seguir albergando la colección en papel y la propia oferta del editor son motivos para no mantener la colección impresa. Por el contrario, aquellos que afirman que mantiene la colección impresa parecen asumir que pasamos por una etapa de transición y que hay aspectos de la edición electrónica que impiden prescindir totalmente del papel (acceso retrospectivo a los fondos, limitación por parte de los editores para ceder los documentos -en préstamos de distinto tipo, etc.).

\section{Satisfac ción con la oferta existente}

Se mide en función de dos variables: el idioma del usuario y su campo de especialización. Las respuestas son claras: hay una satisfacción con las temáticas (así lo señala el 68\%) pero no con el idioma (29\% frente al $71 \%$ que no se considera satisfecho).

Sin embargo, la satisfacción de los usuarios con respecto a los medios electrónicos es tratada en otras preguntas de la encuesta y los resultados permiten matizar algo más estos porcentajes. En términos globales, efectivamente, se puede hablar de satisfacción con respecto a los contenidos de carácter general, aunque algunos usuarios demandan explícitamente más información sectorial; por otra parte, los profesionales también reconocen que para el usuario supone un problema el exceso de publicaciones que hay. En definitiva se puede pensar que los recursos son suficientes, aunque la oferta siempre es mejorable y que el crecimiento de ésta, las actualizaciones, las novedades, etc., generan en el usuario sensación de inundación de información. Ésta es la razón por la que en ocasiones, las respuestas dadas a las preguntas puedan parecen contradictorias.

En cuanto a las respuestas libres los razonamientos contrarios más significativos que dieron los profesionales fueron: la falta de publicaciones electrónicas a texto completo en español y la escasez de recursos en humanidades.

\section{Criterios de selección}

Queda claro que para los profesionales los contenidos (calidad y actualización) priman sobre la tecnología a la hora de decidir la adquisición de recursos electrónicos. 


\section{CALL FOR EDITORS}

\section{New Review of Information and Library Research New Review of Libraries and Lifelong Learning}

Routledge is seeking applications from Intormation Scientists to take up the challenge of editing New Review of Information and Library Research or New Review of Librarles and Lifelong Learning.

The New Revlew of Information and LIbrary Research publishes articles on contemporary information research and how this is shaping present and future intormation and library services.

The New Review of Libraries and Lifelong Learning is designed to report on innovative approaches to providing the various intormation skills and learning environments which toster continuous learning. The journal examines progress in intormation and communication technologies, the re-design of library service delivery, and the scope for libraries to underpin the development of networked learning systems.

The role of editor involves:

- the appointment of an editorial board

- overseeing the peer-review process

- final selection of articles for publication.

Support is given to editors by Routledge's experienced journals publishing staft. This includes:

- financial support for the editor and editorial office

- production and copy-editing support

- marketing support, including promotion to increase readership and copy-flow

- advice and guidance from a managing editor or publisher on all aspects of the publishing process

The ideal candidates will be those with genuine research interests in these fields, who are able to devote time and energy to this project. A clear vision for the future of the journal that the candidate is applying to edit, along with extensive contacts in the field would also be highly desirable. Excellent organisational skills are essential tor these positions. Scholars resident in any country are welcome to apply, as are scholars in the earlier stages of their career.

Applications, for either position, should consist of a CV, covering letter, and statement of editorial intent and should be sent to: Helen Pettet, Taylor \& Francis Ltd, 4 Park Square, Milton Park, Abingdon, Oxtordshire, OX14 4RN, United Kingdom, or by email to: helen.pettet@tandt.co.uk. Tel: +44 (0) 1235828 600; Fax +44 (0) 1235829000 Deadline for applications will be 31st October 2004. 
Curiosamente, el precio, asunto que preocupa mucho en otros puntos del estudio, no resulta un factor decisivo; de hecho ocuparía el cuarto puesto en los criterios de selección, precedido por la claridad de la oferta, lo que parece indicar que se tiene más en cuenta a la hora de seleccionar el "cómo se paga" y "por qué se paga" que "cuánto se paga". Es de suponer que las estructuras de las grandes licencias, con sus formas de calcular los costes de acceso a grandes colecciones, o la aplicación de tarifas basadas en criterios cuantitativos e individualizados de las bibliotecas, creen dudas a la hora de seleccionar uno u otro recurso.

\section{Preferencia en relación con distintos modelos de tarifa}

Los profesionales se inclinan claramente hacia el modelo que permite el uso ilimitado en tiempo y en número de usuarios, tanto para bases de datos como para revistas electrónicas. En segundo lugar se sitúa el pay per view, aunque con porcentajes muy alejados de la primera, y muy cerca de las restantes.

La forma de pago preferente tiene indudables ventajas para los profesionales, si pueden obtener el presupuesto necesario para ello: no tienen que ocuparse de más problemas, aunque normalmente este tipo de tarifas va ligado a direcciones $\mathrm{IP}$, por lo que tienen que disponer de sistemas para que el usuario pueda acceder también desde su domicilio si le está permitida esta forma de trabajo.

El segundo modelo de tarifa preferido bastante alejado en porcentaje del primero, como se ha dicho, es el de pago por documento $(16 \%)$. Las respuestas hacen referencia a que permite el acceso a buenas fuentes de información, pero de uso escaso; se paga sólo lo que se usa; permite controlar mejor el gasto e incluso a alguno le resul-

ta la tarifa más rentable, probablemente porque sus necesidades de información no son elevadas.

Las ventajas de la tarifa por usuario respecto a la tarifa por site, además de hacer referencia a los costes, se relacionan con la posibilidad de que el usuario consulte desde su domicilio y no necesariamente desde la dirección IP.

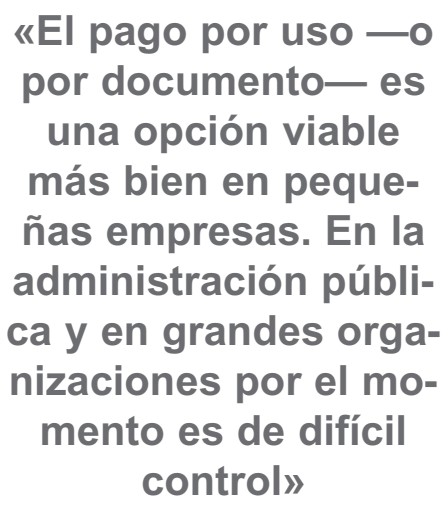

Aunque el modelo de tarifa de pago por documento es la segunda preferencia, la mayoría $(72 \%)$ lo considera poco o nada importante. Sin embargo, para lo poco extendida que está esta forma de pago en las administraciones públicas e incluso en las empresas - por no haber resuelto todavía los problemas administrativos o la desconfianza hacia el pago mediante tarjeta de crédito-, puede considerarse un porcentaje alto, o más bien que se considera una expectativa muy interesante una vez que se resuelvan estas dificultades.

\section{Conclusiones}

1. Los contenidos y la actualización son los criterios de selección más importantes para los profesionales.

2. Los centros de documentación y bibliotecas, con excepción de los de pequeño tamaño, disponen de recursos para resolver sus problemas técnicos.

3. Los profesionales carecen de tiempo para estudiar la documentación relacionada con la oferta de recursos de información, documenta- ción hacia la que, por otra parte, muestran cierta desconfianza.

4. Los profesionales no cuentan con herramientas que les permitan evaluar la utilización de los recursos de que disponen sus centros, lo que sienten como una importante carencia de la que culpan tanto a editores como a agencias.

5. Por lo que se refiere a los recursos, multiplicidad, dispersión (falta de integración) y escasas amigabilidad y usabilidad, siguen percibiéndose como una dificultad para su utilización. Los profesionales piensan que en definitiva a pesar de las inversiones los recursos están infrautilizados.

6. La falta de formación de los usuarios - dicen- es el obstáculo principal para el uso de los recursos de información electrónica, sin que se aprecie ninguna autocrítica en este sentido, pese a que son los más indicados para ayudar a los usuarios a superar este obstáculo.

7. Los grandes centros se inclinan por el modelo de tarifa de acceso ilimitado.

8. El pago por documento es un buen complemento para recursos interesantes pero poco demandados. Para los centros pequeños es además la tarifa más económica.

9. No se aprecian claramente las diferencias entre lo servicios ofrecidos por editores, agencias de suscripciones y distribuidores de bases de datos.

10. Igualmente, no se diferencia entre distintos tipos de recursos: servicios de revistas electrónicas, gateways o bases de datos a texto completo y sus características.

\section{Nota}

El estudio en versión completa estará disponible en un futuro próximo en la sede web de Sedic.

epi@sarenet.es

sedic@sedic.es

info@es.swets.com 